\title{
Effects of antidots on the transport properties of graphene nanoribbons
}

\author{
X. H. Zheng, ${ }^{1}$ G. R. Zhang, ${ }^{1}$ Z. Zeng, ${ }^{1, *}$ Víctor M. García-Suárez, ${ }^{2}$ and Colin J. Lambert ${ }^{2}$ \\ ${ }^{1}$ Key Laboratory of Materials Physics, Institute of Solid State Physics, Chinese Academy of Sciences, Hefei 230031, China \\ ${ }^{2}$ Department of Physics, Lancaster University, Lancaster LA1 4YB, United Kingdom \\ (Received 25 May 2009; revised manuscript received 3 July 2009; published 10 August 2009)
}

\begin{abstract}
Effects of magnetic antidots on the transport properties of zigzag-edged graphene nanoribbons (ZGNRs) are investigated by spin-polarized first-principles calculations combined with a nonequilibrium Green's-function technique. Specifically, the effects of antidots (or holes) with regular shapes (rectangular and triangular) are studied. It is found that rectangular holes with a zero total spin $S_{0}$ and triangular holes with a finite spin $S_{0}$ cause different effects on the equilibrium conductance of ZGNRs. A rectangular hole with zigzag edges parallel to the ribbon edges blocks the transmission of the band edges of both the valence band and the conduction band from both the spin-up channel and the spin-down channel. Thus a much wider transmission gap than the pristine ZGNRs can be observed. However, a triangular hole with zigzag edges blocks transmission from only one spin channel in either the valence-band edge or the conduction-band edge. Thus the gap width in the total conductance is not affected in this case. The difference originates from the different energy shift of the valence band and conduction band relative to Fermi energy as a result of two effects: finite-size effect and spin splitting from the antidot-induced effective internal magnetic field.
\end{abstract}

DOI: $10.1103 /$ PhysRevB.80.075413

PACS number(s): 73.63. $-\mathrm{b}, 72.25 .-\mathrm{b}, 85.65 .+\mathrm{h}$

Graphene nanostructures are supposed to be very important building blocks in future nanoelectronic devices due to their remarkable structural and electronic properties. Among them, graphene nanoribbons (GNRs) have attracted intensive attention ${ }^{1-12}$ and it is found that the properties of GNRs are highly dependent on their sizes and edge shapes. Tightbinding calculations show that armchair-edged GNRs (AGNRs) can be either metallic or semiconducting depending on their widths ${ }^{8,12}$ while first-principles calculations show that AGNRs are always semiconducting with an energy-gap scaling inversely to the GNR width. ${ }^{8-10}$ More interestingly, in the zigzag-edged GNRs (ZGNRs), magnetic ordering is formed due to the unpaired $\pi$ - and $\pi^{*}$-edge electrons. Two most stable spin configurations have been observed: ferromagnetic (FM) and antiferromagnetic (AF), ${ }^{13}$ which mean that the localized edge states on the two sides are FM coupled or AF coupled. Tight-binding calculations show that the ZGNRs are always metallic ${ }^{12}$ while first-principles calculations show that it is metallic only in the FM configuration. There is always an energy gap in the AF configuration. Calculations also show that the most stable configuration is the AF state with the states of different spins in the valence bands and conduction bands localized on different edges while the energy of the states with different spins is degenerate. ${ }^{5,8}$

Meanwhile, quantum dots and antidots made by graphene are another kind of graphene nanostructures which initiate great interests. ${ }^{14-20}$ The dots are just graphene molecules with finite size in all directions while antidots are holes in graphene with some kinds of dots cut away from it. Just like in ZGNRs, magnetism can also be formed in graphene molecules with zigzag edges. ${ }^{15}$ In a graphene molecule with rectangular or hexagonal edges, magnetic moments with the same magnitude and different signs are always formed on the two opposite zigzag edges. Thus the total spin $S_{0}$ in such systems is exactly zero. However, in a molecule with triangular zigzag edges, exactly the same magnetic moments are observed on all edges. So the total spin $S_{0}$ is nonzero. Like in
ZGNRs, the local magnetism in graphene molecules arises from the spin-polarized edge states localized on the zigzag edges.

The local magnetism in graphene nanostructures may qualify graphene nanoribbons and molecules with zigzag edges as promising candidates for application in the spintronic devices. Therefore, the study of spin-polarized transport through these nanostructures are quite interesting. In this work, we build graphene-based devices by combining graphene nanoribbons and antidots with zigzag edges and investigate the electron transport in them. Specifically, we study the effects of graphene antidots (or holes) on the transport properties of ZGNRs. Two kinds of antidots will be considered: rectangular hole and triangular hole.

The model structure is constructed as follows: the scattering region is seamlessly connected to two semi-infinite zigzag $(18,0)$ graphene nanoribbons (following previous convention, it is called as $18-\mathrm{ZGNR}$ ). Both the scattering region and leads have the same width. In the scattering region, a segment of 8-ZGNR ribbon with length of 7 unit cells and an equilateral triangular molecule with edge width of $9 \mathrm{C}$ atoms are cut away from the ribbon (see Fig. 1). All the edge C atoms including those on the ribbon edges and antidot edges are terminated by $\mathrm{H}$ atoms. Note that in the triangle-hole case, in order to saturate the edge $\mathrm{C}$ atoms of the hole by $\mathrm{H}$ conveniently, at each apex of the triangle, one $\mathrm{C}$ atom is put back at its original position [see Fig. 1(b)]. It is well known that graphene-based structures are bipartite lattices which mean that they are composed by two sublattices $(A$ and $B)$. It is found that these three $\mathrm{C}$ atoms at the apexes belong to the same one of the two sublattices. In the calculations, each lead is described by a supercell with four repeated unit cells along $z$ direction and total length of $9.84 \AA$ so that 152 atoms are included in the lead supercell. The length of the scattering region is $51.66 \AA$ which include 708 atoms for the rectangular-hole case and 744 atoms for the triangular-hole case. For simplicity, we call the system with a rectangular hole as "system A" [see Fig. 1(a)] and the system with a 


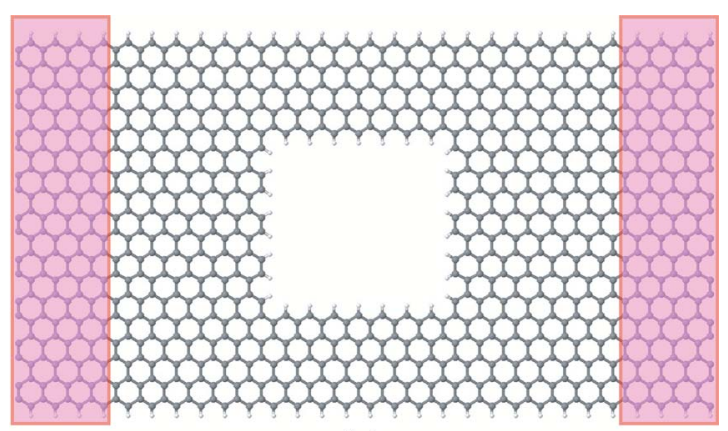

(a)

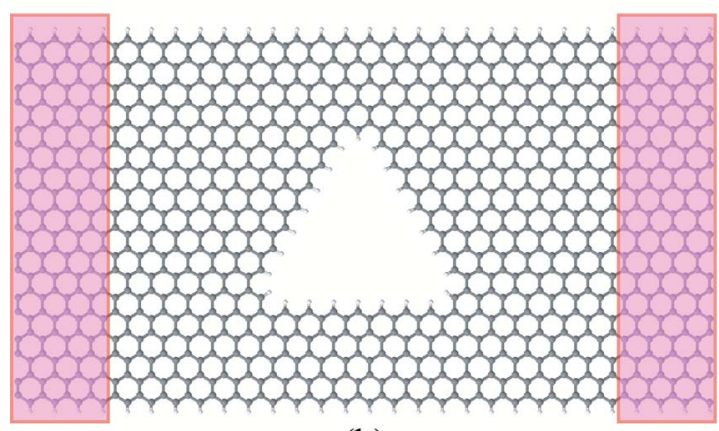

(b)

FIG. 1. (Color online) The geometry structure of the devices. The shadowed areas indicate the electrodes. The region between the shadowed areas is the scattering region: (a) with a rectangular hole (system A); (b) with a triangular hole (system B).

triangular hole as "system B" [see Fig. 1(b)].

All calculations are performed with an ab initio scheme combining density-functional theory (DFT) with the nonequilibrium Green's-function method for quantum transport. The DFT calculation of the lead part is performed with the SIESTA package, ${ }^{21}$ which employs norm-conserving pseudopotentials and linear combinations of atomic orbitals as basis sets. The wave function is expanded with a single-zeta basis set to get a reasonable balance between computation and accuracy. Our test with single-zeta basis set and double-zeta plus polarization basis set in the calculations of band structure of this system shows little difference. This choice was also adopted in the calculations of carbon systems by Louie and co-workers. ${ }^{22}$ The fineness of real-space grid is determined by an equivalent plane-wave cutoff $200 \mathrm{Ry}$. The exchange-correlation potential is treated at the level of localdensity approximation with the form of Perdew-Zunger. ${ }^{23}$ For lead calculations, the Brillouin zone is sampled by a 1 $\times 1 \times 20 k$-point grid while for the scattering region, due to its big size, only $\Gamma$ point is used. Our transport calculations are performed by SMEAGOL program, ${ }^{24-26}$ which interfaces SIESTA and calculates self-consistently the density matrix of the scattering region in the presence of current and external bias by means of nonequilibrium Green's-function technique. The transmission function is calculated by the following Landauer formula: ${ }^{27,28}$

$$
T(E)=\operatorname{Tr}\left[\Gamma_{L}(E) G^{R}(E) \Gamma_{R}(E) G^{A}(E)\right],
$$

where $G^{R / A}$ are the retarded/advanced Green's functions of the scattering region and $\Gamma_{L / R}$ are coupling functions to the left/right leads.

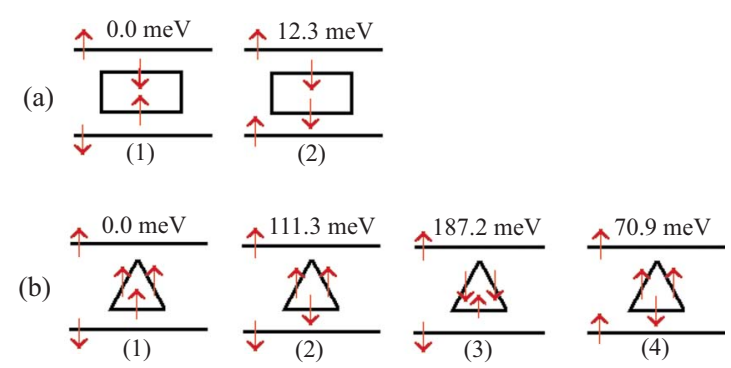

FIG. 2. (Color online) The final stable spin configurations for: (a) system A; (b) system B. The arrows indicate the spins while the number on top of each figure indicates the energy relative to the ground-state configuration in each system.

Before discussion of other properties, all the structures are fully relaxed to reach the force tolerance of $0.04 \mathrm{eV} / \AA$. Both FM and AF initial configurations of the atoms between the two edges are considered and all possible initial spin configurations of the hole edges relative to the ribbon edges are investigated. The final stable spin configurations after full relaxations are shown in Fig. 2. In system A, for either AF or FM state, there are three possible initial spin configurations. We finally get only one stable configuration for both $\mathrm{AF}$ and FM [see (1) and (2) in Fig. 2(a)]. In all other initial spin configurations, spin flip happens at the hole edges and the system reaches the above configurations. In the meantime, we find that the ground state is $\mathrm{AF}$ for the ribbon edges, $\mathrm{AF}$ for the hole edges and $\mathrm{AF}$ for the nearest edges between the ribbon and the hole. Note that for such a wide ribbon, the energy difference between the AF and FM state is small. Likewise, the energy difference between the two stable configurations in Fig. 2(a) is also very small (about $12.3 \mathrm{meV}$ ). In system B, for either AF or FM state, there are four possible initial configurations. For the $\mathrm{AF}$ state, we get three final stable configurations [see (1), (2), and (3) in Fig. 2(b)] while for the FM state, we get only one [see (4) in Fig. 2(b)]. It is interesting to find that in a ZGNR, on one hand, the three edges in the triangle hole are still FM coupled just like in triangular graphene molecules or in triangular antidots in graphene with no edges ${ }^{20}$ on the other hand, the two edges of the ribbon are in AF state, just like in a pristine ZGNR, which is shown by the configuration with the lowest energy [see (1) in Fig. 2(b)]. Moreover, for both system A and system $\mathrm{B}$ in either AF state or FM state, we have considered the zero initial spins for the hole edges. Finite magnetic moments appear on the zigzag edges of the holes finally and the systems reach the configurations shown by (1) and (2) in Fig.

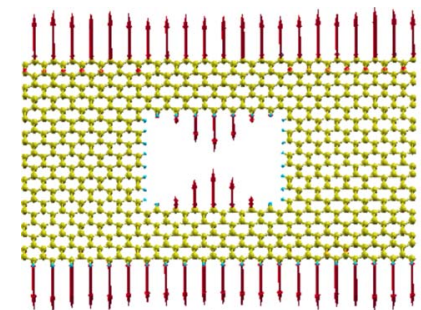

(a)

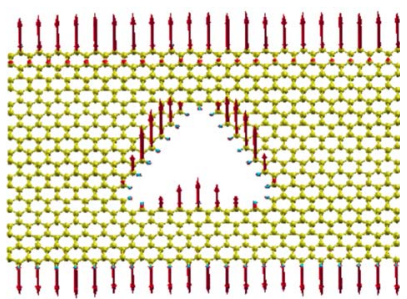

(b)
FIG. 3. (Color online) Magnetic moments on all the atoms in: (a) system A; (b) system B. 

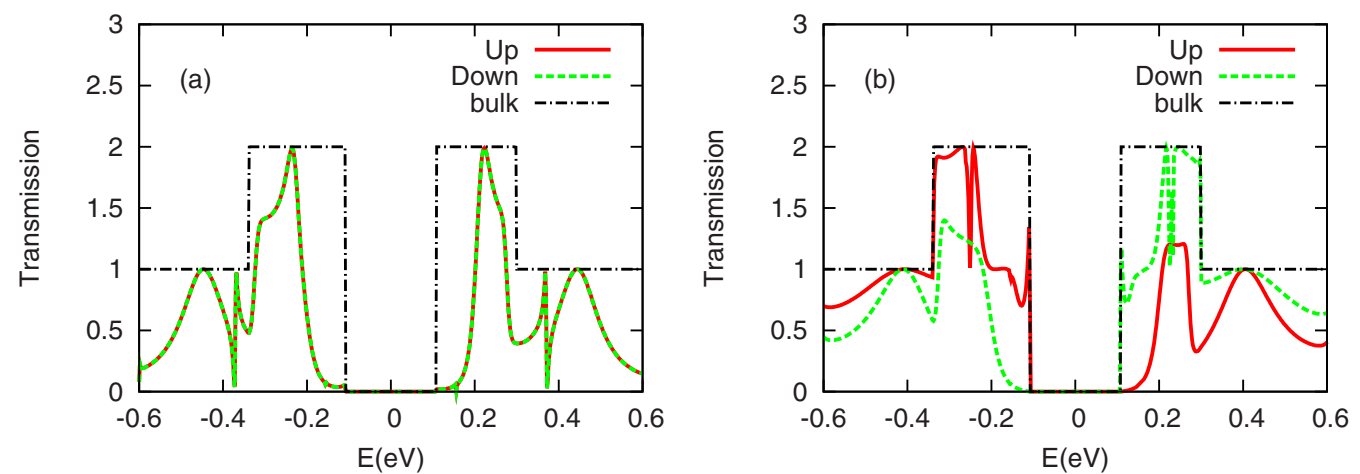

FIG. 4. (Color online) Transmission function of the two systems shown in Fig. 1: (a) for system A; (b) for system B. For comparison, the transmission of the bulk (or pristine) ZGNR is shown by the black dashed lines.

2(a) for system A and those shown by (1) and (4) in Fig. 2(b) for system B. In a word, after full relaxations, we find that, despite the existence of the rectangular or triangular holes, the two ribbon edges are still antiferromagnetically coupled and the magnetic moments on the two ribbon edges are only slightly affected (see Fig. 3).

Furthermore, like in graphene molecules, local magnetic moments are observed on the edges of the holes. The magnetic moments on the ribbon edges and hole edges for the cases with the lowest energies are shown in Fig. 3. In the rectangular hole, the two zigzag edges are antiferromagnetically coupled and the total spin of the hole is zero while in the triangular hole, all three edges are ferromagnetically coupled and the total magnetic moment of the hole is $\sim 6.0 \mu_{B}$. For such bipartite lattices, Lieb's theorem says that the itinerant magnetism is equal to $N_{A}-N_{B}$, where $N_{A}\left(N_{B}\right)$ is the number of atoms on the $A$ sublattice ( $B$ sublattice). ${ }^{29}$ The calculated total magnetic moment in our work agrees exactly with the prediction by the above theorem. Furthermore, the magnetic moment is localized on the edge $\mathrm{C}$ atoms and the contribution on all other atoms is almost negligible.

In the following study of the transport properties of these systems, we will focus on the spin configurations with the lowest energies shown in Fig. 3, in which the ribbon edge states are AF coupled. Since in the AF configuration, ZGNRs are semiconducting and there is a transmission gap around the Fermi level in the pristine ZGNRs (see Fig. 4), we will focus on the effects of the antidots at the top of the valence bands and the bottom of conduction bands. In system A, it is found that the transmission through the band edges of both the valence bands and the conduction bands are completely suppressed [see Fig. 4(a)]. It is natural to see that both the spin-up and spin-down channels are equally suppressed due to the structural and spin symmetry. This will be shown by a much wider transmission gap in the total transmission in system A. However, in system B, the two spin channels are affected quite differently: in the valence bands, only the spindown channel is almost completely suppressed while in the conduction band, only the spin-up channel is almost completely suppressed [see Fig. 4(b)]. To sum up the two spin channels, an unaffected transmission gap can still be observed in the total transmission.

As the transport properties in molecular devices are largely determined by electronic structure of the scattering region, to analyze the origin of the different effects of the rectangular antidot and the triangular antidot, the density of states (DOS) of the scattering region have been studied. We can see the good correspondence between the transmission and the DOS (see Figs. 4-6). Compared with the bulk system, the gap around the Fermi level has been enlarged, which pushes symmetrically outside the valence bands (to lower energy) and the conduction bands (to higher energy) in system A (see Fig. 5). This can be understood by the finite-size effect. In system $\mathrm{A}$, the scattering region consists of two kinds of nanoribbon segments with different width. It is well known that the gap in graphene nanoribbons decreases in-

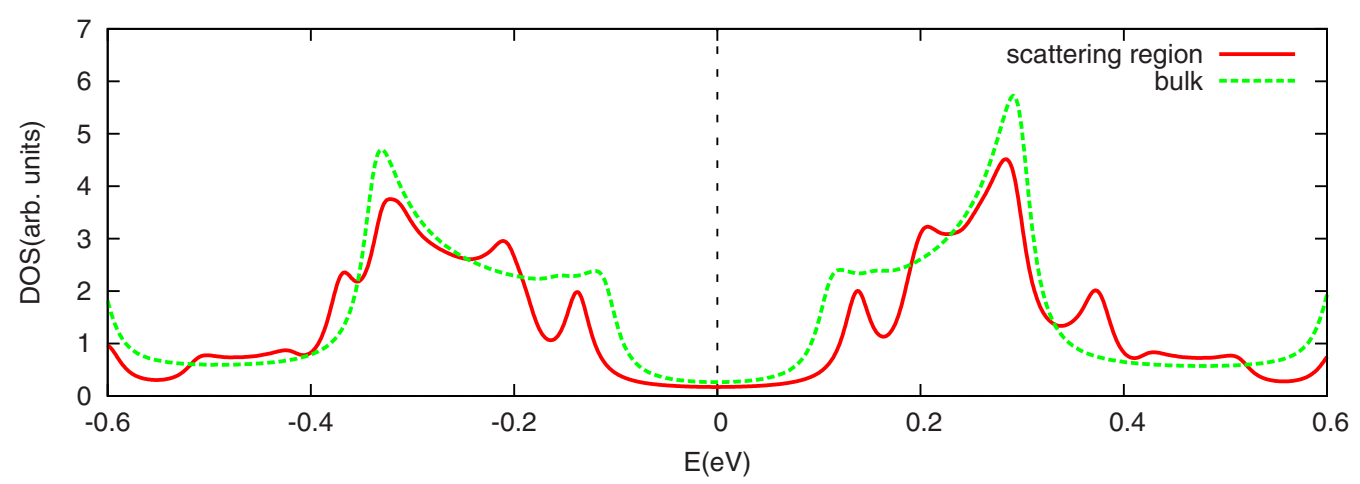

FIG. 5. (Color online) DOS of the two spin channels in system A. Since the two spin channels are degenerate, only one of them is shown. For comparison, the DOS of the bulk or pristine ZGNR is also plotted. The Fermi level is set to that of the lead or the bulk system. 

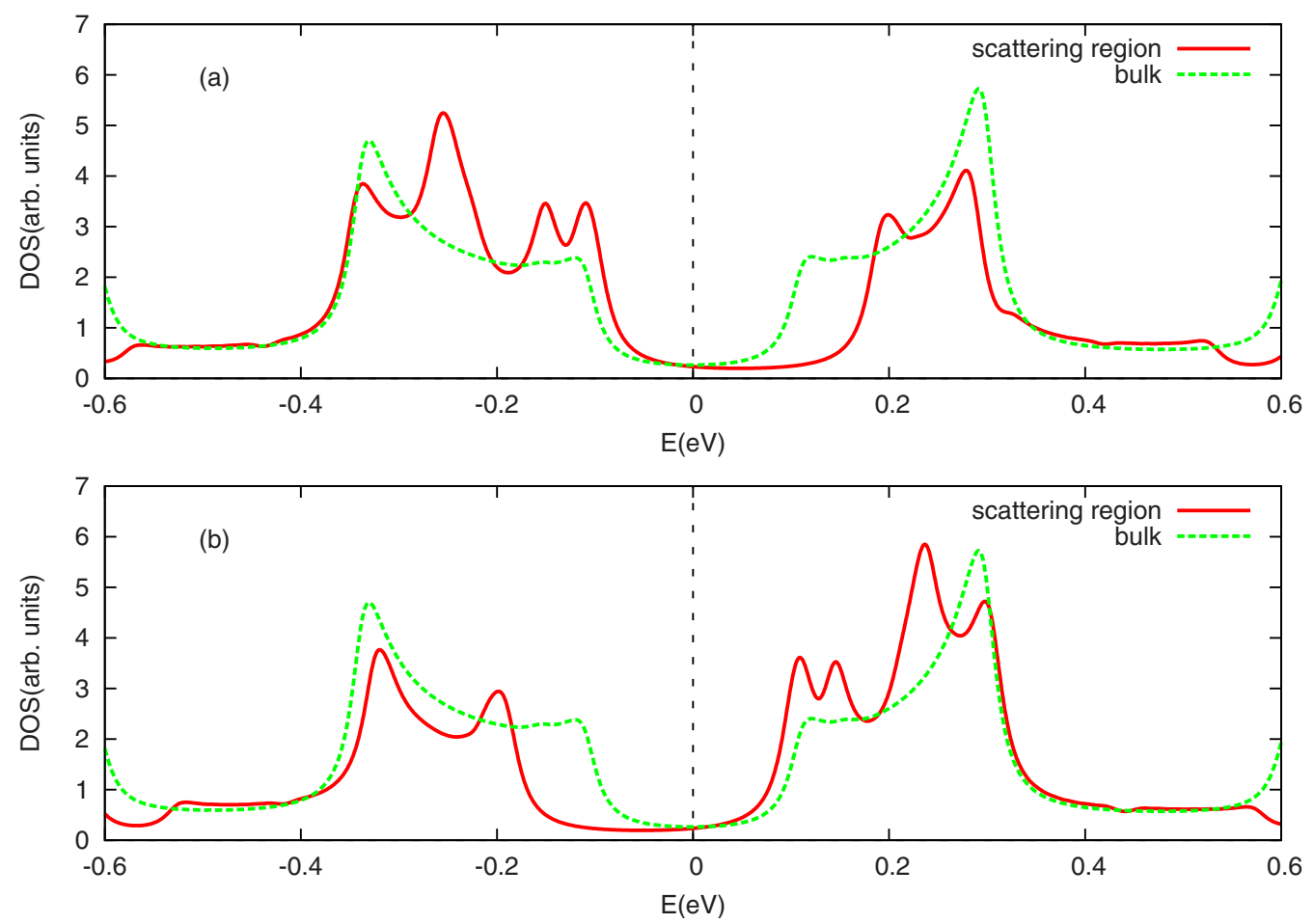

FIG. 6. (Color online) DOS of the two spin channels in system B: (a) for spin-up channel; (b) for spin-down channel. For comparison, the DOS of the bulk or pristine ZGNR is also plotted. The Fermi level is set to that of the lead or the bulk system.

versely to the ribbon width. The two ribbon segments outside the rectangular hole across the ribbon have much smaller width than the other parts of the system and thus have a much larger energy gap. This will enlarge the energy gap of the system to $0.30 \mathrm{eV}$ in the range $[-0.15,0.15] \mathrm{eV}$ when compared with that of the bulk system, about $0.20 \mathrm{eV}$ in the range $([-0.10,0.10] \mathrm{eV})$. This directly results in the suppression of transmission at the band edges of the valence bands and conduction bands. Due to the symmetry of the system, the two spin channels are equally affected.

In contrast, the energy gap has been changed to $0.27 \mathrm{eV}$ in the scattering region in system $B$ (in the energy range $[-0.09,0.18] \mathrm{eV}$ for the spin-up channel and $[-0.18,0.09] \mathrm{eV}$ for the spin-down channel), thus the gap has also been enlarged due to the finite-size effect from the segments around the triangular hole and a much wider conductance gap for each spin channel can be observed (see Fig. $6)$. However, the gap in system B is not symmetrically positioned around the Fermi level, such as in the bulk system and in system A. The states of both the valence bands and conduction bands from the spin-up channel have been shifted to higher energy while those from the spin-down channel have been shifted to lower energy. This actually results in a smaller gap $0.18 \mathrm{eV}$ in the range $[-0.09,0.09] \mathrm{eV}$ in the total DOS, which means that states appear in the gap of the lead. Nevertheless, the leads do not provide transmission channels in the range $[-0.10,-0.09]$ and $[0.09,0.10] \mathrm{eV}$, thus the states in these energy ranges in the scattering region do not contribute to the transmission and the transmission gap in system B still lies in range $[-0.10,0.10] \mathrm{eV}$ with width of $0.20 \mathrm{eV}$, the same as that of the pristine ZGNR.

The above discussion clearly shows that the different shifting directions of the states in the valence bands and conduction bands of the two spin channels give rise to the difference in the transmission. In system A, the states in the valence bands in both the spin channels shift to lower energy while those of the conduction bands from both spin channels shift to higher energy. However, in system B, in both the valence bands and conduction bands, the states from one spin channel all shift in one direction while those from the other spin channel shift in an opposite direction. Therefore, finite-size effect can explain the broadening of the transmission gap in each spin channel but it cannot fully explain the difference of the total transmission gap observed in the two systems. In fact, the difference can be understood by the magnetism of the antidots. In system $\mathrm{B}$, the edge states on the triangular hole are all ferromagnetically coupled and thus a finite total magnetic moment is formed. This finite total magnetism sets up an effective internal magnetic field $\vec{B}$ and cause spin splitting to edge states of the ribbon. The energy change is $\Delta E=-\overrightarrow{\mu_{S}} \cdot \vec{B}=\frac{q}{m} \vec{S} \cdot \vec{B}$, where $\vec{S}$ is the spin of the edge states and $q$ and $m$ are the electron charge and mass. This results in an energy increase for one spin channel and a decrease for the other spin channel. However, in system A, the total magnetic moment induced by the antidot is zero, thus no effective internal magnetic field is created and no energy change from spin splitting is obtained.

Finally, due to the fact that, in system B, at the top of valence bands and the bottom of conduction bands, only one spin channel is conducting, it is highly expected that fully spin-polarized transport can be achieved in this system by applying a gate voltage to shift the Fermi level of the scattering region to the edges of the valence bands or the conduction bands. It may possibly find applications in spintronic devices. 
In summary, we have studied the effects of two kinds of antidots with rectangular shape and triangular shape on the transport properties of ZGNRs. It is found that in the system with rectangular antidot, the transmission gap is enlarged due to the finite-size effect. While in the system with triangular antidot, the spin splitting arising from the effective internal magnetic field induced by the triangular magnetic hole counteracts the finite-size effect and thus the final total transmission gap width is not affected.
This work was supported by the National Science Foundation of China under Grant No 10774148, the special Funds for Major State Basic Research Project of China(973) under Grant No. 2005CB623603, 863 Project, Knowledge Innovation Program of Chinese Academy of Sciences, and Director Grants of CASHIPS. Part of the calculations were performed in Center for Computational Science of CASHIPS and the Shanghai Supercomputer Center.
*Corresponding author; zzeng@theory.issp.ac.cn

${ }^{1}$ K. Wakabayashi, M. Fujita, H. Ajiki, and M. Sigrist, Phys. Rev. B 59, 8271 (1999).

${ }^{2}$ M. Ezawa, Phys. Rev. B 73, 045432 (2006).

${ }^{3}$ D. A. Abanin, P. A. Lee, and L. S. Levitov, Phys. Rev. Lett. 96, 176803 (2006).

${ }^{4}$ S. Okada and A. Oshiyama, Phys. Rev. Lett. 87, 146803 (2001).

${ }^{5}$ Y. W. Son, M. L. Cohen, and S. G. Louie, Nature (London) 444, 347 (2006).

${ }^{6}$ Y. Miyamoto, K. Nakada, and M. Fujita, Phys. Rev. B 59, 9858 (1999).

${ }^{7}$ T. Kawai, Y. Miyamoto, O. Sugino, and Y. Koga, Phys. Rev. B 62, R16349 (2000).

${ }^{8}$ Y. W. Son, M. L. Cohen, and S. G. Louie, Phys. Rev. Lett. 97, 216803 (2006).

${ }^{9}$ V. Barone, O. Hod, and G. E. Scuseria, Nano Lett. 6, 2748 (2006).

${ }^{10}$ C. T. White, J. Li, D. Gunlycke, and J. W. Mintmire, Nano Lett. 7, 825 (2007).

${ }^{11}$ L. Yang, C.-H. Park, Y.-W. Son, M. L. Cohen, and S. G. Louie, Phys. Rev. Lett. 99, 186801 (2007).

${ }^{12}$ K. Nakada, M. Fujita, G. Dresselhaus, and M. S. Dresselhaus, Phys. Rev. B 54, 17954 (1996).

${ }^{13}$ T. B. Martins, R. H. Miwa, A. J. R. da Silva, and A. Fazzio, Phys. Rev. Lett. 98, 196803 (2007).

${ }^{14}$ M. Ezawa, Phys. Rev. B 76, 245415 (2007).

${ }^{15}$ J. Fernandez-Rossier and J. J. Palacios, Phys. Rev. Lett. 99,
177204 (2007).

${ }^{16}$ M. Ezawa, Eur. Phys. J. B 67, 543 (2009).

${ }^{17}$ T. G. Pedersen, C. Flindt, J. Pedersen, N. A. Mortensen, A.-P. Jauho, and K. Pedersen, Phys. Rev. Lett. 100, 136804 (2008).

${ }^{18}$ T. G. Pedersen, C. Flindt, J. Pedersen, A.-P. Jauho, N. A. Mortensen, and K. Pedersen, Phys. Rev. B 77, 245431 (2008).

${ }^{19}$ D. Yu, E. M. Lupton, M. Liu, W. Liu, and F. Liu, Nano Res. 1, 56 (2008).

${ }^{20}$ L. Chen, D. Yu, and F. Liu, Appl. Phys. Lett. 93, 223106 (2008).

${ }^{21}$ J. M. Soler, E. Artacho, J. D. Gale, A. Garcia, J. Junquera, P. Ordejon, and D. Sanchez-Portal, J. Phys.: Condens. Matter 14, 2745 (2002).

${ }^{22}$ Y.-W. Son, J. Ihm, M. L. Cohen, S. G. Louie, and H. J. Choi, Phys. Rev. Lett. 95, 216602 (2005).

${ }^{23}$ J. P. Perdew and A. Zunger, Phys. Rev. B 23, 5048 (1981).

${ }^{24}$ A. R. Rocha, V. Garcia-Suarez, S. W. Bailey, C. J. Lambert, J. Ferrer, and S. Sanvito, Nature Mater. 4, 335 (2005).

${ }^{25}$ A. R. Rocha, V. Garcia-Suarez, S. W. Bailey, C. J. Lambert, J. Ferrer, and S. Sanvito, Phys. Rev. B 73, 085414 (2006).

${ }^{26}$ I. Rungger and S. Sanvito, Phys. Rev. B 78, 035407 (2008).

${ }^{27}$ S. Datta, in Electronic Transport in Mesoscopic Systems, edited by H. Ahmed, M. Pepper, and A. Broers (Cambridge University Press, Cambridge, England, 1995).

${ }^{28}$ H. Haug and A.-P. Jauho, Quantum Kinetics in Transport and Optics of Semiconductors (Springer-Verlag, Berlin, 1996).

${ }^{29}$ E. H. Lieb, Phys. Rev. Lett. 62, 1201 (1989). 